\title{
Automonitoração e Seguimento de Regras Nutricionais em Diabetes: Dois Estudos de Caso ${ }^{1}$
}

\author{
Mariene da Silva Casseb \\ Michele da Silva Malcher Bispo \\ Eleonora Arnaud Pereira Ferreira
}

Universidade Federal do Pará

\begin{abstract}
RESUMO
A literatura demonstra que obesidade associada ao diabetes impõe dificuldades ao controle glicêmico. Estudos sugerem que adultos com diabetes apresentam maior dificuldade de adesão à dieta, quando comparada à prática regular de atividade física e ao uso de medicamento. Por outro lado, pesquisas apontam que a utilização de registros de automonitoração auxilia adultos com doenças crônicas a seguirem as instruções prescritas por profissionais de saúde. Este estudo investigou os efeitos do uso de registros de automonitoração no seguimento de regras nutricionais por duas mulheres adultas com diagnóstico de diabetes e obesidade, atendidas no ambulatório de um hospital universitário. Realizaram-se dezenove visitas domiciliares a cada participante utilizando-se roteiros de entrevista e protocolos de registro de automonitoração. Os dados obtidos foram analisados segundo três categorias descritivas (refeições realizadas com adesão, refeições realizadas sem adesão e refeições não realizadas) e em relação a três etapas de automonitoração (sem planejamento, com planejamento e de manutenção). Os resultados sugerem que o uso de automonitoração, acompanhado de treino em análise do comportamento alimentar, mostrou-se eficiente para o aumento da adesão às regras nutricionais.
\end{abstract}

Palavras-chave: diabetes; automonitoração; adesão à dieta.

\begin{abstract}
Self-management and adherence to diet in diabetes: two case studies

Literature has shown that obesity associated to diabetes imposes difficulties to glycemic control. Studies suggest that adult patients with diabetes present greater difficulty to diet adherence when compared to physical activity and medication. However, studies indicate that self-monitoring and the use of recordings help adults with chronic diseases adhere to diets and follow instructions prescribed by health professionals. The present study investigated the effects of self monitoring and compliance to diet rules on two obese adult females diagnosed with diabetes attending a university hospital. Nineteen home visits were made to each patient where they were interviewed following schedules and self monitoring register protocols. Data collected was assessed according to three descriptive categories (meal with adherence, no adherence and no meal) and three stages of self-monitoring (no planning, planning and maintenance). The results suggest that self-monitoring followed by a training in eating behaviors proved to be effective in increasing adherence to nutritional rules.
\end{abstract}

Keywords: diabetes; self-monitoring; adherence to diet.

A obesidade é avaliada como um problema de saúde pública e sabe-se que sua incidência e controle dependem de uma complexa interação de variáveis biológicas, comportamentais e culturais (Duchesne, 1998; Márquez-Ibañez, Armendáriz-Anguiano, BacardíGascón \& Jiménez-Cruz, 2008). A obesidade pode ser definida como a condição na qual o indivíduo apresenta quantidade excessiva de gordura corporal e o aumento do peso é resultado do acúmulo da gordura no tecido adiposo. Este acúmulo pode ocorrer devido a uma ingestão calórica maior que o gasto energético, o que está diretamente ligado ao sedentarismo e a variáveis sociodemográficas (Baretta, Baretta, Peres \& 2007; Cunha, Peixoto, Jardim \& Alexandre, 2008; Keher \& cols., 2007). 
É provável que, anualmente na América Latina, 200.000 pessoas morram de doenças relacionadas à obesidade. Entre estas doenças está o diabetes, cuja prevalência é maior em pessoas com Índice de Massa Corpórea (IMC) acima de 28 (Coutinho, 2001). Dentre estas pessoas, a dieta tem sido apontada como a maior dificuldade para a adesão ao tratamento. Estudos também sugerem que fatores como regras extensas e complexas, baixa assertividade e dificuldades em discriminar níveis glicêmicos por meio de sintomas, podem estar associados à baixa adesão à dieta e, consequentemente, ao tratamento do diabetes (Brandão, 2003; Ferreira, 2001).

Para Heller e Kerbauy (2000), o comportamento alimentar deve ser analisado como aprendido e sua reeducação deve ser feita por meio de técnicas de autocontrole e de uma análise funcional individualizada, na qual podem ser investigados os vários fatores associados à ingestão alimentar do paciente. Neste sentido, um programa de treinamento em autocontrole de comportamento alimentar tem como benefício permitir ao indivíduo manter o controle do próprio comportamento e seguir as orientações do tratamento sempre que for necessário, a partir da análise dos ganhos obtidos. Estudos sugerem que estes ganhos podem ser produzidos pelo uso de procedimentos de automonitoração envolvendo protocolos de registro de comportamento alimentar (Coutinho, 2001; Duchesne \& cols., 2007; Ferreira, 2001; Ferreira \& Casseb, 2002; Nansel \& cols., 2007; Peyrot \& Rubin, 2007).

Neste caso, o uso de princípios da análise do comportamento mostra-se útil em estudos que visem à intervenção, pois, por meio da análise funcional do comportamento, torna-se possível identificar as contingências externas ao organismo das quais seu comportamento é função (Skinner, 1998/1953) e definir procedimentos - tanto pela equipe profissional quanto pelo paciente - que serão mais adequados para se chegar ao controle do peso.

Dentre os vários modelos teóricos que dão ênfase à análise funcional como proposta de intervenção, destaca-se o modelo construcional de Israel Goldiamond (Goldiamond, 1974), que tem como base a construção e ampliação de repertórios mais funcionais, sem focalizar a eliminação de comportamentos. De acordo com este modelo, a construção e a ampliação de repertórios se fazem por meio de aproximações sucessivas (modelagem), a partir de comportamentos adequados que o paciente já apresenta ao início do atendimento, até que ele amplie seu repertório comportamental anali- sando as contingências que o mantêm e aproximandoo do objetivo (comportamento-meta).

Um procedimento bastante utilizado no modelo construcional é a automonitoração, pois permite a observação, avaliação e intervenção comportamental, além de fornecer dados para a condução de análises funcionais, delimitação dos objetivos de intervenção, planejamento e avaliação dos resultados (Bohm \& Gimenes, 2008). Estudos mostram que procedimentos de automonitoração têm sido eficazes na construção de repertórios de comportamentos de adesão ao tratamento por indivíduos com doenças crônicas (Brandão, 2003; Casseb, 2005; Chaves \& Oyama, 2007; Ferreira, 2001; Ferreira \& Casseb, 2002; Malcher, 2005; Oliveira, Ferreira \& Casseb, 2005; West, DiLillo, Bursac, Gore \& Greene, 2007). Segundo Tobin, Reynolds, Holroyd e Creer (1986), automonitoração refere-se a observações que o indivíduo faz do próprio comportamento por meio do registro de fatores que afetam o seu tratamento e consequentemente a sua saúde.

A psicologia aplicada à área de saúde utiliza princípios da análise do comportamento para auxiliar na prevenção, no diagnóstico, no tratamento e na reabilitação de pessoas com doenças crônicas. Nesta abordagem, o enfoque do trabalho do psicólogo na saúde é analisar contingências, ou seja, os eventos antecedentes ao comportamento, o comportamento em si e os eventos consequentes que mantém a doença e os que promovem a saúde. É necessário que a intervenção seja ajustada às necessidades e peculiaridades do paciente e a sua realidade (Amaral, 1999; Guimarães, 1999; Malerbi, 2000). Esse ajustamento pode se dar por meio da investigação da linha de base do comportamento do paciente referente ao manejo da doença, para que se avaliem quais são os comportamentos adequados que o paciente já apresenta, e assim promover a generalização para outros comportamentos saudáveis de forma gradativa. O paciente, portanto, pode observar cada ganho obtido.

Segundo os princípios da análise do comportamento, uma resposta que tenha sido aprendida sob certas contingências de reforço e que tenha sido submetida a um processo de extinção (redução da frequência da resposta mediante a retirada da consequência reforçadora) pode, em uma situação futura, ser restabelecida em condições semelhantes àquela na qual foi adquirida (Keller, 1973). Assim, a problemática da adesão ao tratamento médico sugere que pro- 
gramas de intervenção atentem para a questão do reforço intermitente necessário à manutenção dos comportamentos adequados já instalados.

A obesidade e o diabetes são doenças crônicas que exigem o seguimento de regras complexas referentes à alimentação, atividade física e uso de medicamento. $\mathrm{O}$ seguimento destas regras exige um conjunto de mudanças no estilo de vida do paciente, o que muitas vezes dificulta a adesão ao tratamento (Ferreira, 2001). Com base em princípios da análise do comportamento e no modelo construcional, este estudo verificou os efeitos do uso de automonitoração sobre o seguimento de regras nutricionais por duas mulheres adultas com diabetes e obesidade.

\section{MÉTODO}

\section{Participantes}

Participaram deste estudo duas mulheres adultas, inscritas em um programa de atenção multiprofissional à pessoa com diabetes de um hospital universitário. Ambas apresentavam dificuldades no seguimento das instruções fornecidas pelo Serviço de Nutrição (50\% ou menos de refeições em um dia realizadas de acordo com as orientações nutricionais), com diferentes histórias de treino em automonitoração de comportamento alimentar. A Participante 1 (P1) não possuía histórico de treino, enquanto a Participante 2 (P2) já havia participado de estudo anterior no qual foi submetida a um treino em registros de comportamento alimentar (Ferreira, 2001). As principais características das participantes ao início do estudo estão descritas na Tabela 1 .

TABELA 1

Características Sociodemográficas e Estado Clínico das Participantes ao Início do Estudo

\begin{tabular}{|c|c|c|}
\hline Variáveis & Participante 1 & Participante 2 \\
\hline Idade & 67 anos & 63 anos \\
\hline Escolaridade & $\begin{array}{l}\text { Ensino fundamental incompleto } \\
\qquad\left(4^{a} \text { série }\right)\end{array}$ & $\begin{array}{l}\text { Ensino fundamental incompleto } \\
\qquad\left(5^{\mathrm{a}} \text { série }\right)\end{array}$ \\
\hline Ocupação & Aposentada/Dona de casa & Aposentada/Dona de casa \\
\hline Estrutura familiar & $\begin{array}{l}\text { Solteira } \\
\text { Cuidadora de um sobrinho de } 10 \text { anos de } \\
\text { idade }\end{array}$ & $\begin{array}{l}\text { Casada } \\
\text { Morando com esposo, dois filhos, um } \\
\text { genro e uma neta. }\end{array}$ \\
\hline Renda familiar & 1 salário mínimo & $3^{1 / 2}$ salários mínimos \\
\hline Tipo de DM & Tipo 2 & Tipo 2 \\
\hline Tempo de diagnóstico & 10 anos & 5 anos \\
\hline Outras patologias & $\begin{array}{c}\text { Obesidade Classe I } \\
\text { Artrose e proeminência óssea nas vérte- } \\
\text { bras }\end{array}$ & $\begin{array}{l}\text { Obesidade Classe I } \\
\text { Hipertensão }\end{array}$ \\
\hline Uso de medicação & $\begin{array}{l}\text { Anti-inflamatório hormonal } \\
\text { Hipoglicemiante oral }\end{array}$ & Hipotensores \\
\hline
\end{tabular}

\section{Instrumentos utilizados}

(a) Roteiro de entrevista inicial: usado para coleta de dados sociodemográficos das participantes, seus conhecimentos sobre diabetes e seu histórico de obesidade.

(b) Roteiro de entrevista final: usado para coletar informações sobre a participação no estudo, analisar os resultados alcançados e a generalização dos ganhos obtidos. (c) Protocolo nutricional: material impresso oferecido pelo Serviço de Nutrição, contendo orientações sobre alimentos recomendados e alimentos de uso restrito, incluindo sugestão de fracionamento e a quantidade de cada alimento sugerida por refeição.

(d) Protocolo de registro de automonitoração semanal da alimentação: usado para caracterizar a linha de base do comportamento alimentar de cada participante; composto por seis colunas correspon- 
dentes a seis dias de uma semana, nas quais a participante deveria anotar os alimentos ingeridos em cada refeição/dia; o primeiro registro era feito em parceria com a terapeuta-pesquisadora (TP) durante entrevista realizada em ambulatório.

(e) Protocolo de registro diário da alimentação: contendo colunas correspondentes ao horário da refeição, tipo de alimento e quantidade ingerida, incluindo o registro da ingestão de alimentos entre as principais refeições; eram entregues à participante tantos protocolos quantos fossem os dias correspondentes ao intervalo entre as entrevistas.

(f) Protocolo de registro de planejamento diário da alimentação: usado para planejar previamente a alimentação diária da participante, contendo coluna para o registro do planejamento das refeições do dia seguinte à entrevista, coluna para o registro das refeições efetivamente realizadas (incluindo a descrição de horário, qualidade e quantidade de alimentos ingeridos), e coluna para avaliação da correspondência entre o planejado e o executado, incluindo o contexto em que as refeições ocorreram.

(g) Protocolo de registro de manutenção do seguimento das regras nutricionais: usado para a participante avaliar se estava seguindo as regras nutricionais e mantendo comportamentos alimentares adequados, composto por colunas correspondentes às refeições/dia, em número igual aos dias do intervalo entre as entrevistas, no qual a participante deveria marcar com um $X$ as refeições realizadas de acordo com as orientações do serviço de nutrição, segundo sua autoavaliação.

(h) Prontuário do paciente: documento onde são registrados, em ordem cronológica, todos os procedimentos realizados com o paciente durante seu acompanhamento no hospital.

\section{Procedimento de Coleta de Dados}

Foram realizadas com cada participante dezenove entrevistas gravadas em áudio, sendo uma em ambulatório e dezoito em ambiente domiciliar, seguindo seis etapas.

Etapa 1 - Identificação dos critérios de inclusão. Nesta etapa foram realizadas: (a) entrevista com o Serviço de Nutrição do programa para estabelecimento de parceria e orientação sobre os critérios de encaminhamento de pacientes ao Serviço de Psicologia; (b) análise de prontuário dos pacientes encaminhados e obtenção do protocolo nutricional estabele- cido para cada participante após consulta nesta especialidade; (c) entrevista com pacientes encaminhados para obtenção do consentimento livre e esclarecido, seguindo as normas da Resolução 196/1996-Conselho Nacional de Saúde; e (d) aplicação do Roteiro de Entrevista Inicial.

Etapa 2 - Caracterização da linha de base. Fez-se o levantamento da linha de base de comportamentos alimentares de cada participante, utilizando-se o protocolo de registro de automonitoração semanal da alimentação; obteve-se também a descrição das regras nutricionais pela participante para verificação do seu entendimento sobre as mesmas.

Etapa 3 - Treino em automonitoração de comportamentos correspondentes às regras nutricionais. Inicialmente, cada participante recebeu instruções sobre o preenchimento do protocolo de registro diário da alimentação. A seguir, durante as entrevistas realizadas em domicílio da participante em dias alternados, a TP realizava em conjunto com a participante a análise do preenchimento do protocolo, comparando o registro com as regras do protocolo nutricional.

Etapa 4 - Treino em automonitoração com planejamento. Cada participante recebeu instruções sobre o preenchimento do protocolo de registro de planejamento diário da alimentação; durante as entrevistas realizadas em dias alternados, a TP fazia em conjunto com a participante a análise do preenchimento do protocolo, comparando o registro dos comportamentos planejados com o dos comportamentos executados, tendo como referência as regras dispostas no protocolo nutricional; fez-se também análise do contexto em que as refeições eram realizadas.

Etapa 5 - Acompanhamento dos registros de automonitoração com avaliação dos resultados alcançados. Foram realizadas análises dos registros obtidos com o protocolo de registro de manutenção do seguimento das regras nutricionais até que a participante alcançasse o critério mínimo de $50 \%$ das refeições diárias realizadas de acordo com as regras nutricionais; a cada entrevista, fazia-se o preenchimento do registro diário da alimentação referente ao dia anterior à visita domiciliar, para análise da manutenção da adesão às regras nutricionais.

Etapa 6 - Avaliação dos resultados alcançados. Utilizou-se o roteiro de entrevista final e fez-se análise sobre os resultados obtidos com a participação no estudo. Nesta etapa também se fez análise dos registros em prontuários, realizados durante a condução deste estudo. 


\section{Organização e Análise dos Dados}

Os dados coletados foram organizados por participante com vistas à análise intra-sujeito e posteriormente à análise entre sujeitos. As entrevistas foram transcritas e os relatos foram organizados tomando-se como eixos de análise as descrições das regras nutricionais e a autoavaliação da adesão ao tratamento. Quanto aos dados obtidos por meio de protocolos de automonitoração, os registros foram analisados levando-se em consideração as orientações contidas no protocolo nutricional de cada participante. $\mathrm{Na}$ análise da correspondência entre o que era sugerido no protocolo e o que era registrado pela participante, contou-se com a colaboração de nutricionistas do programa no qual as pacientes estavam inscritas. Os registros eram analisados e, a cada correspondência adequada, era atribuído um ponto de acerto para o registro da paciente, de modo que, a cada registro, para um total de seis refeições, a participante poderia obter até seis pontos, o que corresponderia a $100 \%$ de acertos. Em seguida, os resultados obtidos com os registros de automonitoração foram organizados de acordo com três categorias descritivas: refeições realizadas com adesão, refeições realizadas sem adesão e refeições não realizadas. Estas categorias foram analisadas de acordo com as etapas de coleta de dados, incluindo análises em etapas de automonitoração sem planejamento (referentes às Etapas 2 e 3 da coleta de dados), automonitoração com planejamento (Etapa 4) e automonitoração de manutenção (Etapa 5).

\section{RESULTADOS}

A entrevista inicial realizada na Etapa 1 confirmou o critério de dificuldade no seguimento das regras nutricionais em mais de 50\% das refeições/dia, necessário para que as pacientes fossem incluídas no estudo.

A Tabela 2 apresenta a correspondência entre a descrição das orientações nutricionais feita pelas participantes e as orientações contidas no protocolo nutricional fornecido pelo Serviço de Nutrição ao início do estudo.

TABELA 2

Correspondência Entre as Orientações Nutricionais Fornecidas Pelo Serviço de Nutrição e a Descrição Destas Pelas Participantes

\begin{tabular}{|c|c|c|c|}
\hline \multirow[t]{2}{*}{ Refeições } & \multirow[t]{2}{*}{ Orientações contidas no protocolo } & \multicolumn{2}{|c|}{ Descrição das orientações por participante } \\
\hline & & Participante 1 & Participante 2 \\
\hline Desjejum & $\begin{array}{c}2 \text { colheres de leite desnatado } \\
1 \text { pão careca ou } 4 \text { cream cracker. } \\
1 \text { colher de chá rasa de margarina. }\end{array}$ & $\begin{array}{l}\text { três torradas ou cinco bolachas cream } \\
\text { cracker }\end{array}$ & $\begin{array}{c}\text { pouco café com leite molico, } \\
\text { torradas ou cream cracker e } \\
\text { um pão careca sem miolo. }\end{array}$ \\
\hline Lanche 1 & $\begin{array}{c}2 \text { porções de fruta ou } \\
1 \text { copo pequeno de suco ou } \\
2 \text { biscoitos cream cracker. }\end{array}$ & um suco de fruta ou uma fruta. & $\begin{array}{c}\text { uma banana, uma laranja, } \\
\text { uma maçã. Qualquer fruta que } \\
\text { não tenha gordura. }\end{array}$ \\
\hline Almoço & $\begin{array}{c}4 \text { colheres de arroz } \\
1 \text { colher rasa de farinha } \\
1 / 2 \text { bife de carne } \\
1 \text { porção de fruta } \\
1 \text { colher de óleo } \\
\text { (evitar fritura) }\end{array}$ & $\begin{array}{l}\text { três colheres de arroz ou macarrão; } \\
\text { peixe ou carne grelhada. }\end{array}$ & $\begin{array}{c}\text { bife grelhado, arroz escorrido; } \\
\text { duas colheres de macarrão; } \\
\text { galinha assada ou guisada; } \\
\text { uma concha de feijão apenas } \\
\text { uma vez na semana; } \\
\text { canja. }\end{array}$ \\
\hline Lanche 2 & $\begin{array}{c}2 \text { porções de fruta ou } \\
1 \text { copo de suco ou } \\
2 \text { biscoitos cream cracker }\end{array}$ & O mesmo do lanche da manhã. & $\begin{array}{l}\text { um copo de suco de fruta ou } \\
\text { uma fruta. }\end{array}$ \\
\hline Jantar & $\begin{array}{c}4 \text { colheres de arroz } \\
4 \text { colheres de frango desfiado ou } \\
2 \text { pedaços pequenos de carne } \\
1 \text { colher de óleo } \\
\text { (evitar fritura) }\end{array}$ & $\begin{array}{l}\text { Jantar comida leve, não me encharcar } \\
\text { de carne vermelha. }\end{array}$ & $\begin{array}{l}\text { Mais leve. Reduzir a quantida- } \\
\text { de do almoço, apesar de ser o } \\
\text { mesmo prato; uma banda de } \\
\text { mamão ou um suco. }\end{array}$ \\
\hline Ceia & $\begin{array}{c}\text { Chá ou } \\
\text { um copo pequeno de leite desnatado. }\end{array}$ & $\begin{array}{l}\text { Não relatou, pois não executava esta } \\
\text { refeição. }\end{array}$ & $\begin{array}{l}\text { um copo de leite com bolacha } \\
\text { cream cracker. }\end{array}$ \\
\hline
\end{tabular}


Observou-se que ambas as participantes demonstram entendimento de que as refeições devem ser fracionadas, realizadas em horários regulares e na sequência estabelecida pelo serviço de nutrição, embora a descrição não seja feita com precisão quanto ao tipo e à quantidade de alimentos ingeridos. A descrição apresentada pela participante 2 demonstra melhor detalhamento do que a descrição apresentada pela participante 1, provavelmente como decorrência da história prévia de treinamento.

Os resultados obtidos por meio da análise dos protocolos de registro de automonitoração estão demonstrados na Figura 1.

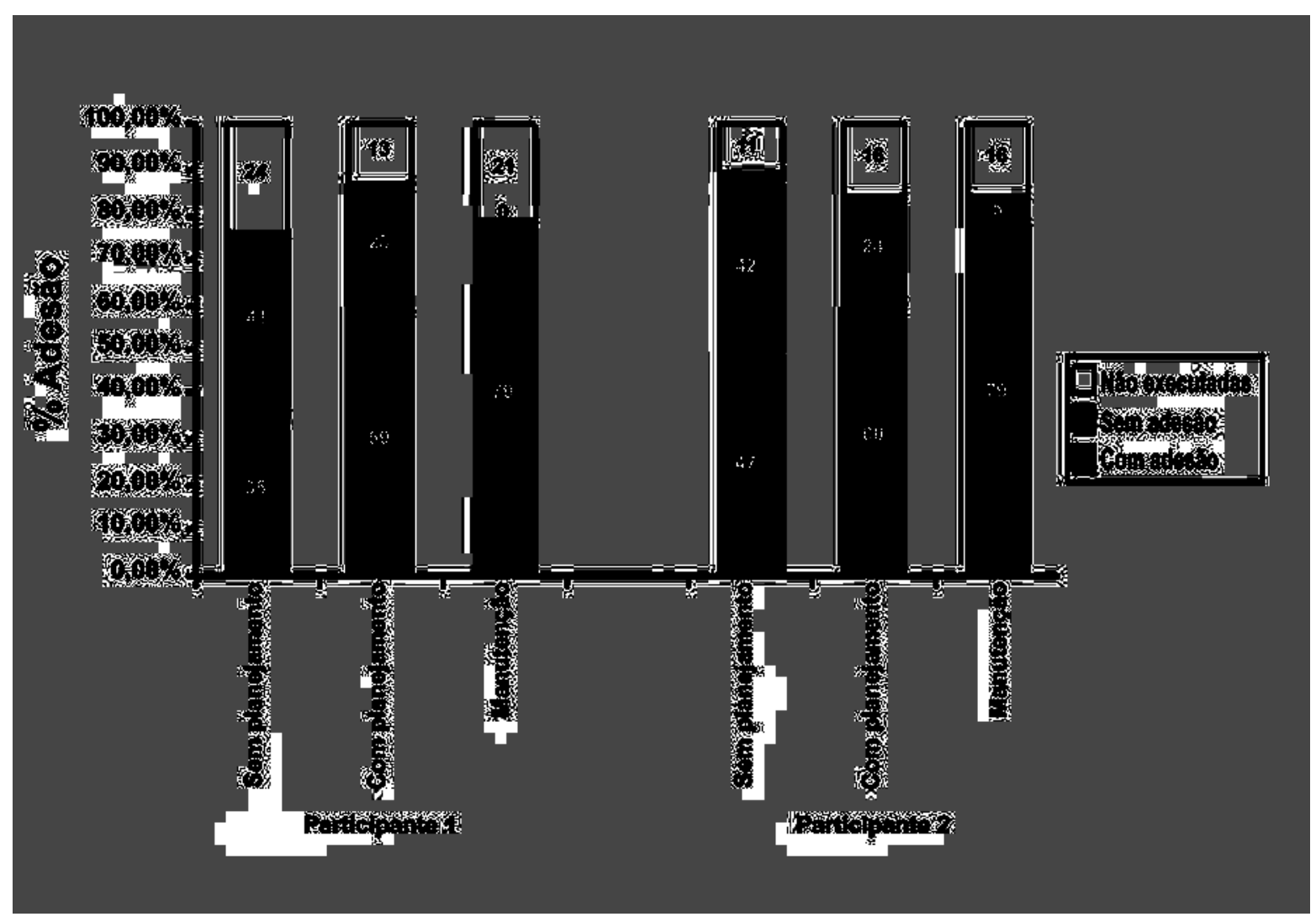

Figura 1. Percentual de refeições realizadas com adesão, sem adesão e não executadas pelas Participantes 1 e 2 durante as etapas de automonitoração sem planejamento, com planejamento e de manutenção.

Observou-se que as duas participantes já apresentavam, ao início do estudo, comportamentos alimentares adequados durante a etapa de automonitoração sem planejamento, apresentando $35 \%$ (participante 1) e $47 \%$ (participante 2) de refeições executadas com adesão. Entretanto, os valores não chegavam a 50\%, demonstrando não adesão às regras nutricionais de acordo com os critérios estabelecidos para este estudo.

Durante a etapa de automonitoração com planejamento, as duas participantes alcançaram o critério estabelecido para adesão. No caso da participante 1, nota-se aumento de refeições realizadas com adesão (de 35 para 59\%), redução de refeições realizadas sem adesão (de 41 para 28\%) e redução de refeições não realizadas (de 24 para 13\%). No caso da participante
2 , verificou-se aumento de refeições realizadas com adesão (de 47 para 60\%), redução de refeições realizadas sem adesão (de 42 para $24 \%$ ) e discreto aumento de refeições não realizadas (de 11 para 16\%).

Quanto aos resultados obtidos na etapa de automonitoração de manutenção, observou-se que a participante 1 apresentou aumento de refeições realizadas com adesão (de 59 para 79\%), redução até eliminação de refeições realizadas sem adesão (de 28 para $0 \%$ ) e aumento de refeições não realizadas (de 13 para $21 \%$ ). A participante 2 obteve aumento de refeições realizadas com adesão (de 60 para $79 \%$ ), redução de refeições realizadas sem adesão (de 24 para 5\%) e manutenção do percentual de refeições não realizadas $(16 \%)$. 
Os registros obtidos com os protocolos também permitiram a análise da adesão das participantes a cada tipo de refeição, conforme está apresentado na Figura 2.

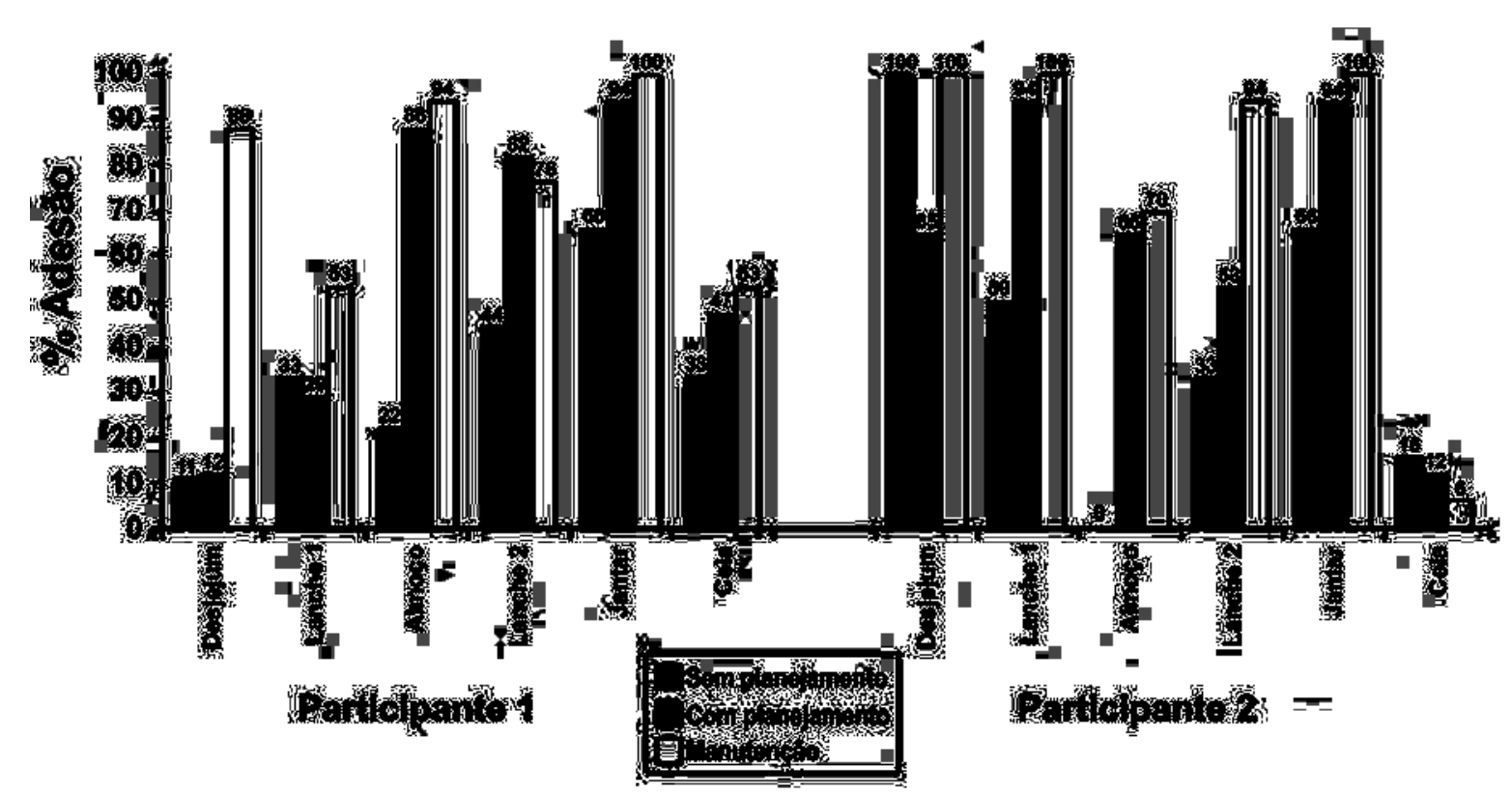

Figura 2. Percentual de adesão das Participantes 1 e 2, por tipo de refeição, durante as etapas de automonitoração do comportamento alimentar.

Dentre as refeições executadas com adesão durante a etapa de automonitoração sem planejamento, verificou-se que a participante 1 apresentou maior adesão no jantar $(66 \%)$. A participante 2 apresentou total adesão no desjejum (100\%) e 50\% de adesão no lanche 1 e no jantar. Nas outras refeições, as participantes apresentaram adesão abaixo de $50 \%$, o que se caracteriza dificuldade no seguimento das regras nutricionais nessas refeições.

Durante a etapa de automonitoração com planejamento, verificou-se que a participante 1 permaneceu apresentando maior adesão no jantar (94\%), melhorando sua adesão no almoço $(88 \%)$ e no lanche 2 $(82 \%)$. As refeições que obtiveram menor adesão foram o desjejum e o lanche 1 . No caso da participante 2, a refeição executada com maior adesão correspondeu ao lanche 1 (94\%). O desjejum, o almoço e o jantar foram realizados com $65 \%$ de adesão cada um. Por outro lado, esta participante não apresentou melhora na realização da ceia, mantendo percentual baixo (12\%), como ocorria antes do treino (16\%). As demais refeições foram realizadas com adesão superior a $50 \%$.
Ao longo da etapa de automonitoração de manutenção, pode-se observar que a participante 1 apresentou adesão acima de $50 \%$ em todas as refeições. Verificou-se também que esta participante apresentou maior adesão no jantar (100\%), almoço (94\%), desjejum (88\%) e lanche $2(76 \%)$. Mesmo com menor adesão dentre as refeições (53\%), verifica-se que o lanche 1 e a ceia apresentaram aumento nos índices de adesão quando comparados com a etapa anterior. No caso da participante 2 , verifica-se que o desjejum, lanche 1 e o jantar apresentaram adesão de $100 \%$. A ceia foi a única refeição com percentual abaixo do critério de $50 \%$, apresentando apenas $6 \%$ de adesão.

$\mathrm{Na}$ análise dos registros feitos pela equipe multiprofissional em prontuário de cada participante, deu-se destaque aos níveis glicêmicos e ao peso corporal apresentados pelas participantes durante o período da coleta de dados deste estudo. $\mathrm{O}$ índice glicêmico das participantes foi medido por glicemia de jejum, realizada no laboratório de análises clínicas do hospital universitário. A Tabela 3 apresenta os registros de glicemia e peso das participantes 1 e 2 no decorrer do estudo. 
TABELA 3

Glicemia de Jejum e Peso das Participantes 1 e 2, Segundo Registros no Prontuário no Decorrer do Estudo

\begin{tabular}{ccccccccc}
\hline \multicolumn{10}{c}{ PARTICIPANTE 1 } \\
\hline Datas & $30 / 08$ & $15 / 09$ & $25 / 09$ & $29 / 09$ & $03 / 10$ & $08 / 10$ & $17 / 11$ & $05 / 12$ \\
Glicemia & 92 & 129 & 120 & 91 & 95 & 112 & 99 & 105 \\
& $\mathrm{mg} / \mathrm{dl}$ & $\mathrm{mg} / \mathrm{dl}$ & $\mathrm{mg} / \mathrm{dl}$ & $\mathrm{mg} / \mathrm{dl}$ & $\mathrm{mg} / \mathrm{dl}$ & $\mathrm{mg} / \mathrm{dl}$ & $\mathrm{mg} / \mathrm{dl}$ & $\mathrm{mg} / \mathrm{dl}$ \\
Peso & 86 & 86 & 85,900 & 85,900 & 85,850 & 85,650 & 85,450 & 84,900 \\
& $\mathrm{~kg}$ & $\mathrm{~kg}$ & $\mathrm{~kg}$ & $\mathrm{Kg}$ & $\mathrm{kg}$ & $\mathrm{kg}$ & $\mathrm{kg}$ & $\mathrm{kg}$ \\
\hline Datas & $30 / 08$ & $17 / 09$ & $20 / 09$ & $09 / 10$ & $10 / 10 /$ & $24 / 10$ & $08 / 11$ & $07 / 12$ \\
\hline \multirow{2}{*}{ Glicemia } & 82 & 145 & \multirow{2}{*}{ sem registro } & sem registro & 146 & 130 & 120 & 110 \\
& $\mathrm{mg} / \mathrm{dl}$ & $\mathrm{mg} / \mathrm{dl}$ & 82,200 & 82,200 & $\mathrm{mg} / \mathrm{dl}$ & $\mathrm{mg} / \mathrm{dl}$ & $\mathrm{mg} / \mathrm{dl}$ & $\mathrm{mg} / \mathrm{dl}$ \\
Peso & 84 & sem registro & $\mathrm{kg}$ & $\mathrm{Kg}$ & sem registro & sem registro & 82,000 & 82,200 \\
& $\mathrm{~kg}$ & & & & & $\mathrm{~kg}$ & $\mathrm{~kg}$ \\
\hline
\end{tabular}

A comparação entre os resultados apresentados nas Figuras 1 e 2 com os apresentados na Tabela 3 sugerem uma correspondência entre o aumento no percentual de comportamentos de adesão às regras nutricionais, identificada a partir da análise dos registros de automonitoração e a estabilidade no estado clínico apresentada pelas duas participantes no decorrer deste estudo. No caso da participante 1 , observou-se redução do peso corporal (de 86 para $84,900 \mathrm{~kg}$ ) e estabilidade do nível glicêmico em valores considerados como normais, mesmo com a suspensão do hipoglicemiante oral prescrito pela médica do programa após sucessivos resultados bem sucedidos de controle glicêmico.

No caso da participante 2 , também se observa manutenção de níveis glicêmicos considerados dentro da normalidade para a idade da participante e o tempo de diagnóstico. Os resultados também mostraram perda discreta de peso pela participante 2 .

A Tabela 4 apresenta os relatos das duas participantes referentes aos demais componentes do programa de tratamento - atividade física e uso de medicação - ao final do estudo.

TABELA 4

Relatos das Participantes 1 e 2 Referentes à Atividade Física e à Medicação

\begin{tabular}{|c|c|c|}
\hline \multirow{2}{*}{ CATEGORIAS } & \multicolumn{2}{|c|}{ RELATOS DAS PARTICIPANTES } \\
\hline & PARTICIPANTE 1 & PARTICIPANTE 2 \\
\hline $\begin{array}{l}\text { Referentes à } \\
\text { atividade física }\end{array}$ & $\begin{array}{l}\text { Olha, eu até me sinto mais leve [após iniciar } \\
\text { as caminhadas regulares], eu fico menos } \\
\text { cansada... Já vou lá na padaria que é no } \\
\text { final da rua; devagar, mas não me sinto } \\
\text { mal. Antes eu nem ía... } \\
\text { Levo o R. pro colégio, volto, vou na feirinha } \\
\text { comprar o almoço; tudo devagar por causa } \\
\text { da coluna... }\end{array}$ & $\begin{array}{l}\text { Caminhada? Tem semana que eu não faço, } \\
\text { mas tem semana que eu caminho. } \\
\text { Eu caminhei ontem, por mais ou menos meia } \\
\text { hora. Quero ver se caminho sábado de novo! } \\
\text { Ainda caminhei indo pro Banco. Lá do Banco } \\
\text { que eu vim pegar ônibus para voltar. Ontem } \\
\text { caminhei até lá na farmácia! } \\
\text { Eu caminhei sexta, domingo e ontem. Um dia } \\
\text { sim, um dia não. }\end{array}$ \\
\hline $\begin{array}{l}\text { Referentes à } \\
\text { medicação }\end{array}$ & $\begin{array}{l}\text { Eu tenho pavor que o meu diabetes chegue } \\
\text { a trezentos e lá vai [...] e ainda ter um } \\
\text { coma, Deus me livre... } \\
\text { Eu já pedi, mas a médica disse que não vai } \\
\text { mais me dar o remédio porque eu não } \\
\text { preciso mais... Eu tenho medo de ficar sem } \\
\text { o remédio, mas a doutora diz: 'a senhora } \\
\text { não precisa porque a senhora se cuida...' } \\
\text { Aí eu tô sem o remédio. }\end{array}$ & $\begin{array}{l}\text { Eu não estou tomando medicamento, porque } \\
\text { a doutora falou pra mim que o meu diabetes } \\
\text { não precisava de remédio. É só fazer controle. }\end{array}$ \\
\hline
\end{tabular}


No caso da participante 1 observa-se a instalação de atividade física leve (caminhar até a feira e outros locais próximos) e a suspensão do hipoglicemiante oral. Pode ser percebida, também, a dificuldade desta participante em aceitar a retirada deste medicamento, mesmo com a autorização da médica.

A participante 2 apresentou mudanças na prática de atividade física, pois voltou a realizar caminhadas com duração de 30 minutos, em dias alternados, tal como já havia ocorrido em treino anterior. Como a paciente não possuía prescrição de hipoglicemiante oral, nenhuma modificação foi identificada neste aspecto.

\section{DISCUSSÃO}

O estudo apresentou resultados positivos em relação ao uso de automonitoração do comportamento alimentar na promoção de seguimento das orientações nutricionais pelas duas participantes. Neste sentido, a análise do comportamento, utilizando o modelo construcional como base, foi de grande relevância, permitindo o treino em automonitoração de acordo com as características individuais e com a realidade de cada participante, conforme sugere a literatura (Duchesne, 1998; Ferreira, 2001; Goldiamond, 1974; Guimarães, 1999).

Apesar de não ter sido identificada melhora no fracionamento das refeições, visto que o percentual de refeições não realizadas sofreu pouca alteração com a introdução do planejamento, houve um aumento significativo na execução de refeições adequadas durante a condição de manutenção, quando comparada à automonitoração sem planejamento. Desta forma, o estudo demonstrou que as participantes apresentaram melhores índices de adesão às orientações nutricionais a partir do uso de registros de automonitoração de seus comportamentos alimentares, confirmando estudos anteriores (Brandão, 2003; Ferreira, 2001; Ferreira \& Casseb, 2002; Malcher, 2005; Tobin \& cols., 1986). Identificou-se, também, aumento na adesão à atividade física e suspensão da prescrição do hipoglicemiante oral usado pela participante 1 , em virtude do controle glicêmico atingido, sugerindo ganhos secundários ao uso de registros de automonitoração do comportamento alimentar.

A dificuldade de adesão da participante 2 em relação à ceia verificada na automonitoração de manutenção (Figura 2) pode ser analisada como consequência da inadequação desta refeição à rotina da paciente, devido à proximidade entre o horário do jantar e o horário de dormir. Isto corrobora a necessidade de se avaliar as idiossincrasias para o estabelecimento do tratamento a fim de que este não se torne oneroso, dificultando a adesão do paciente (Duchesne, 1998; Ferreira, 2001; Guimarães, 1999).

Os ganhos obtidos confirmam a importância da introdução gradativa das regras para o adequado gerenciamento de doenças crônicas, obedecendo ao modelo construcional na construção e ampliação de comportamentos de adesão, de acordo com o repertório inicial e a linha de base do comportamento-alvo que o paciente apresenta ao início do tratamento (Ferreira, 2001; Goldiamond, 1974).

Observou-se que o uso de procedimentos de automonitoração produz benefícios, mas não é sinônimo de $100 \%$ de adesão, pois o paciente pode abandonar o tratamento, ou parte dele, em determinadas situações como foi o caso da participante 2, que havia descontinuado a prática de atividade física introduzida em treinamento comportamental anterior. No entanto, a literatura mostra que o automonitoração favorece a adesão ao tratamento permitindo que o paciente avalie os ganhos obtidos e retorne ao tratamento sempre que haja necessidade (Duchesne, 1998; Heller \& Kerbauy, 2000). O rápido restabelecimento de comportamentos alimentares mais adequados pela participante 2 , que ao início do estudo se apresentavam com índices inferiores a $50 \%$ apesar da história prévia de treinamento em automonitoração, e o retorno à prática de atividade física confirmam os princípios da análise do comportamento que enfatizam o aumento de frequência de repertórios treinados previamente quando diante de contingências de reforço semelhantes àquelas nas quais estes foram instalados (Keller, 1973).

Este resultado também ressalta a relevância de se manter reforçadores sociais para comportamentos correspondentes às instruções apresentados pelo paciente, além daqueles reforçadores intrinsecamente relacionados ao comportamento de adesão, de modo a garantir a manutenção dos ganhos com a intervenção. Para isso, torna-se indispensável a inclusão de atividades periódicas de automonitoração e "re-treino" na rotina do paciente, para favorecer a recuperação e a manutenção da adesão ao tratamento de doenças crônicas, visto que a recaída em não adesão tem mostrado alta probabilidade de ocorrência em tratamentos de longo prazo (Ferreira, 2001; Malerbi, 2000).

A partir de orientações para resolução de problemas e do planejamento prévio das refeições, as participantes passaram a optar por alimentos mais adequados ao tratamento e a controlar melhor a quantidade 
de alimentos ingeridos. Neste sentido, a situação financeira se mostra como uma variável que exerce influência direta sobre o comportamento alimentar. Fatores econômicos como baixa renda financeira, má administração do dinheiro, déficit na análise de custos na escolha dos alimentos a serem comprados, favorecem o consumo de alimentos contraindicados, levando à baixa adesão à dieta. $\mathrm{O}$ planejamento das refeições com base na análise do contexto estabelecido permite a reformulação das contingências, de maneira que produzam o comportamento alimentar esperado para a adesão à dieta, de acordo com a realidade e as possibilidades do paciente, confirmando a literatura que sugere a necessidade de individualização nas orientações de tratamento (Malerbi, 2000).

Convém ressaltar que a aplicação do treino de automonitoração deve ser estabelecida de forma gradual, por meio de registros com diferentes níveis de complexidade, iniciando o treino com registros mais simples para que o paciente não sinta dificuldades e se familiarize, motivando-se para o tratamento. Em seguida são introduzidos registros mais complexos, nos quais o paciente detalha seu comportamento alimentar permitindo-lhe analisar as contingências que controlam seu comportamento, instalar o repertório de autocontrole e, consequentemente, minimizar ou até mesmo extinguir comportamentos prejudiciais à saúde e à adesão ao tratamento. Quando o paciente se torna capaz de analisar contingências e exerce o autoconhecimento, pode-se voltar a introduzir registros simples em que o paciente avalie os ganhos em manter comportamentos alimentares adequados ao tratamento (Ferreira, 2001; Heller \& Kerbauy, 2000).

$\mathrm{O}$ apoio da equipe multiprofissional e da rede social são fatores fundamentais para que o paciente garanta a manutenção dos comportamentos adequados obtidos com a intervenção. Desse modo, expõe-se a necessidade de se estruturar programas de atendimento a pacientes crônicos que considerem as especificidades da população atendida, os recursos comportamentais de que já dispõe, voltando-se prioritariamente para prevenção e educação em saúde, assim como para a melhoria da qualidade de vida.

\section{REFERÊNCIAS}

Amaral, V. L. A. R. (1999). Novos desafios na formação do psicólogo na área da saúde. Em R. R. Kerbauy (Org), Comportamento e saúde: Explorando alternativas (pp. 3-9). Santo André, SP: ArBytes.

Baretta, E., Baretta, M., \& Peres, K. G. (2007). Nível de atividade física e fatores associados em adultos no Município de Joaçaba,
Santa Catarina, Brasil. Cadernos de Saúde Pública, 23(7), 1595-1602.

Bohm, C. H., \& Gimenes, L. S. (2008). Automonitoramento como técnica terapêutica e de avaliação comportamental. Revista Psicolog, 1(1), 88-100.

Brandão, W. L. O. (2003). Adesão ao tratamento por portadores de diabetes Tipol e Tipo 2: Efeitos do treino de discriminação de dicas internas e externas. Dissertação de mestrado nãopublicada, Programa de Pós-Graduação em Teoria e Pesquisa do Comportamento, Universidade Federal do Pará, Belém.

Casseb, M. S. (2005). Prevenção em diabetes: Efeitos do treino de automonitoração na redução de fatores de risco. Dissertação de mestrado não-publicada, Programa de Pós-Graduação em Teoria e Pesquisa do Comportamento, Universidade Federal do Pará, Belém, PA.

Chaves, E. C., \& Oyama, S. M. R. (2007). Abordagem telefônica como estratégia para promoção da saúde. Revista Gaúcha de Enfermagem, 28(2), 171-179.

Coutinho, W. (2001) La obesidad como factor de riesgo de enfermidades y condiciones asociadas. Consenso Latino Americano em Obesidade. Retirado em março de 2001, de http://www. abeso.org.br/consenso.br

Cunha, I. C., Peixoto, M. R. G., Jardim, P. C. B. V., \& Alexandre, V. P. (2008). Fatores associados à prática de atividade física na população adulta de Goiânia: Monitoramento por meio de entrevistas telefônicas. Revista Brasileira de Epidemiologia, $11(3), 495-504$.

Duchesne, M. (1998). Tratamento cognitivo-comportamental da obesidade. Em A. Halpern, A. F. G. Matos, H. L. Suplicy, M. G. Mancini \& M. T. Zanella (Orgs.), Obesidade (pp. 261-273). São Paulo: Lemos Editorial.

Duchesne, M., Appolinário, J. C., Rangé, B. P., Freitas, S., Papelbaum, M., \& Coutinho, W. (2007). Evidências sobre a terapia cognitivo-comportamental no tratamento de obesos com transtorno da compulsão alimentar periódica. Revista de Psiquiatria do Rio Grande do Sul, 29(1), 80-92.

Ferreira, E. A. P. (2001). Adesão ao tratamento em portadores de diabetes: Efeitos de um treino em análise de contingências sobre comportamentos de autocuidado. Tese de doutorado nãopublicada, Universidade de Brasília.

Ferreira E. A. P., \& Casseb M. S. (2002). Efeitos do uso de registros de automonitoração no seguimento de regras nutricionais por uma portadora de diabetes tipo 2 e obesidade classe I. Diabetes Clínica, 6(6), 452-459.

Goldiamond I. (1974). Toward a construcional approach to social problems: Ethical and constitutional issues raised by applied behavior analysis. Behaviorism, 2, 1-84.

Guimarães S. S. (1999). Psicologia da saúde e doenças crônicas. Em R. R. Kerbauy (Org.), Comportamento e saúde: Explorando alternativas (pp. 22-45). Santo André, SP: ArBytes.

Heller, D. C. L., \& Kerbauy R. R. (2000). Redução de peso: Identificação de variáveis e elaboração de procedimentos com uma população de baixa renda e escolaridade. Revista Brasileira de Terapia Comportamental e Cognitiva, 2(1), 31-52.

Keher, G. M., Souza, V. F. M., Vágula, S., Fiorese, L.V., Nardo Júnior, N., \& Pereira, V. R. (2007). Prevenção e tratamento da 
obesidade: Indicativos do sul do Brasil. Ciência, Cuidado e Saúde, 6(2), 427-432.

Keller F. S. (1973). Aprendizagem: Teoria do reforço. (R. Azzi, L. O. Zimmerman, \& S. Queiroz, Trads.). São Paulo: EPU.

Malcher, M. S. (2005). Efeitos do treino de cuidadores sobre comportamentos de apoio à adesão às orientações nutricionais por portadores de diabetes. Dissertação de mestrado nãopublicada, Programa de Pós-Graduação em Teoria e Pesquisa do Comportamento, Universidade Federal do Pará, Belém.

Malerbi, F. E. K. (2000). Adesão ao tratamento. Em R. R. Kerbauy (Org.), Sobre comportamento e cognição: Vol. 5 - Psicologia comportamental e cognitiva, conceitos, pesquisa e aplicação, a ênfase no ensinar, na emoção e no questionamento clínico (pp. 148-155). Santo André, SP: ArBytes.

Márquez-Ibañez, B., Armendáriz-Anguiano, A. L., BacardíGascón, M., \& Jiménez-Cruz, A. (2008). Revisión de ensayos clínicos controlados mediante cambios en el comportamiento para el tratamiento de la obesidad. Nutrição Hospitalar, 23(1), $1-5$.

Nansel, T. R., Iannotti, R. J., Simons-Morton, B.G., Cox, C., Plotnick, L. P., Clark, L. M., \& Zeitzoff, L.(2007). Diabetes personal trainer outcomes: Short-term and 1-year outcomes of a diabetes personal trainer intervention among youth with type 1 diabetes. Diabetes Care, 30(10), 2471-2477.

Oliveira, L. C. C., Ferreira, E. A. P., \& Casseb, M. S. (2005). Os efeitos do uso de registros de automonitoração sobre a mode- lagem de comportamentos alimentares adequados em pacientes com risco para desenvolver diabetes. Retirado em março de 2006, de www.cultura.ufpa.br/rcientifica/melhores_trabs_05/ed 06_lcco.pdf

Peyrot, M., \& Runbin, R. R. (2007). Behavioral and psychosocial interventions in diabetes: A conceptual review. Diabetes Care, 30(10), 2433-2440.

Skinner, B. F. (1998). Ciência e comportamento humano (10 ed.) (J. C. Todorov \& R. Azzi, Trads.). São Paulo: Martins Fontes. (Original publicado em 1953).

Tobin, D. L., Reynolds, R.V.C., Holroyd, K. A., \& Creer, T. L. (1986). Self-management and social learning theory. Em K. A. Holroyd, \& T. L. Creer (Orgs.), Self-management of chronic disease: Handbook of clinical interventions and research (pp. 269-303). Orlando, FL: Academic Press.

West, D. S., DiLillo, V., Bursac, Z., Gore, S. A., \& Greene, P. G. (2007). Motivational interviewing improves weight loss in women with type 2 diabetes. Diabetes Care, 30(5), 1081-1087.

Recebido: 14/01/2008

Última revisão: $02 / 12 / 2008$ Aceite final: 10/12/2008

\section{Nota:}

${ }^{1}$ As autoras agradecem à equipe do Programa de Atenção Multidisciplinar ao Portador de Diabetes, do Hospital Bettina Ferro de Souza, Universidade Federal do Pará, em especial às nutricionistas Eunice Amaral e Mary Barros, pelo apoio à realização deste estudo. Pesquisa parcialmente financiada pelo CNPq por meio do Edital MCT/CNPq 50-2006 (Processo 401815/2007-2)

\section{Sobre as autoras:}

Mariene da Silva Casseb: Doutoranda no Programa de Pós-Graduação em Teoria e Pesquisa do Comportamento, Universidade Federal do Pará, bolsista CAPES. Endereço eletrônco: casseb4@yahoo.com.br.

Michele da Silva Malcher Bispo: Mestre em Teoria e Pesquisa do Comportamento pela Universidade Federal do Pará, Psicóloga da Secretaria Estadual de Saúde do Estado do Pará. Endereço eletrônco: mimalcher@ yahoo.com.br.

Eleonora Arnaud Pereira Ferreira: Doutorado em Psicologia pela Universidade de Brasília, Professora Orientadora do Programa de Pós-Graduação em Teoria e Pesquisa do Comportamento, Universidade Federal do Pará. Endereço eletrônco: eleonora@ufpa.br.

Endereço para correspondência: Rua Augusto Corrêa, 1, Universidade Federal do Pará, Cidade Universitária Professor José da Silveira Netto - Instituto de Filosofia e Ciências Humanas - Faculdade de Psicologia, Guamá - 66075-900 Belém - PA. 